\title{
Malassezia infection associated with chronic spontaneous urticaria without angioedema: a report on five cases
}

\author{
Vijay Zawar ${ }^{1}$, Manoj Pawar ${ }^{1 凶}$, Shrikant Kumavat ${ }^{1}$
}

\begin{abstract}
Introduction: Chronic spontaneous urticaria (CSU) is a challenging condition to treat and it significantly affects quality of life. Bacterial, viral, parasitic, and fungal infections have been associated with triggering and/or perpetuating urticaria in certain individuals. There is a paucity of literature on CSU associated with Malassezia infection.

Methods and results: We present a case series of five patients with CSU without angioedema in whom we observed temporal association of Malassezia infection with CSU. The presence of Malassezia was confirmed by clinical examination, Wood's lamp, and $\mathrm{KOH}$ examination. The patients with CSU experienced improvement after specific antifungal therapy.

Conclusion: Malassezia infection may be associated with recurrent and chronic urticaria in a certain group of susceptible patients and thus specific targeted therapy against it might result in complete remission of urticaria along with clearing of the infection.
\end{abstract}

Keywords: Malassezia, chronic urticaria, association, antifungals

Received: 14 September 2017 | Returned for modification: 23 November 2017 | Accepted: 5 December 2017

\section{Introduction}

Chronic spontaneous urticaria (CSU) is defined as the occurrence of itchy wheals, angioedema, or both over a 6-week period independent of external stimuli (1). It is thought to have a more significant impact on patients' quality of life than any other allergic disease because it affects the activity of daily life and causes sleep disturbances, emotional problems, loss of energy, work absenteeism, and frustration in social relations (2). Activation of cutaneous mast cells, which contain preformed mediators such as histamine, plays a major role in the pathogenesis of urticaria. Many etiological factors have been implicated in the pathogenesis of CSU, including bacterial and viral infections, parasites, fungi, food and food additives, and autoantibodies directed against IgE receptors. Fungal infections are a less-recognized and lessreported cause of urticaria. There are isolated reports mentioning Candida and dermatophytes in association with urticaria (1, 3, 4). Tang et al. (5) observed the role of Malassezia furfur in the prevalence of chronic urticaria among a ship's crew. We report a case series of five patients with CSU without angioedema possibly induced by Malassezia infection.

\section{Methods and results}

Five patients with CSU were identified with coexisting Malassezia infection: four males and one female (median age: $32 \mathrm{yrs,} \mathrm{range:}$ 16-40 yrs). The clinical data for the patients are summarized in Table 1. Investigations such as complete blood cell count, ESR, CRP, blood sugar, urine and stool analysis, liver, renal, and thyroid function test, anti-thyroid peroxidase antibodies, anti-thyroglobulin, autologous serum skin test, HIV antibody, autoantibodies (ANA, ds-DNA), and IgE were either normal or negative. The Helicobacter pylori profile and latex-IgE were negative. Serum tryptase and $\mathrm{C}_{3}$, C4 levels could not be studied due to a lack of resources.

All five patients presented with recurrent pruritic, evanescent, erythemato-edematous papules and plaques of prolonged duration ( $>6$ weeks) with fluctuating severity of symptoms suggestive of CSU with a significant compromise to their quality of life (Figs. 1-5).
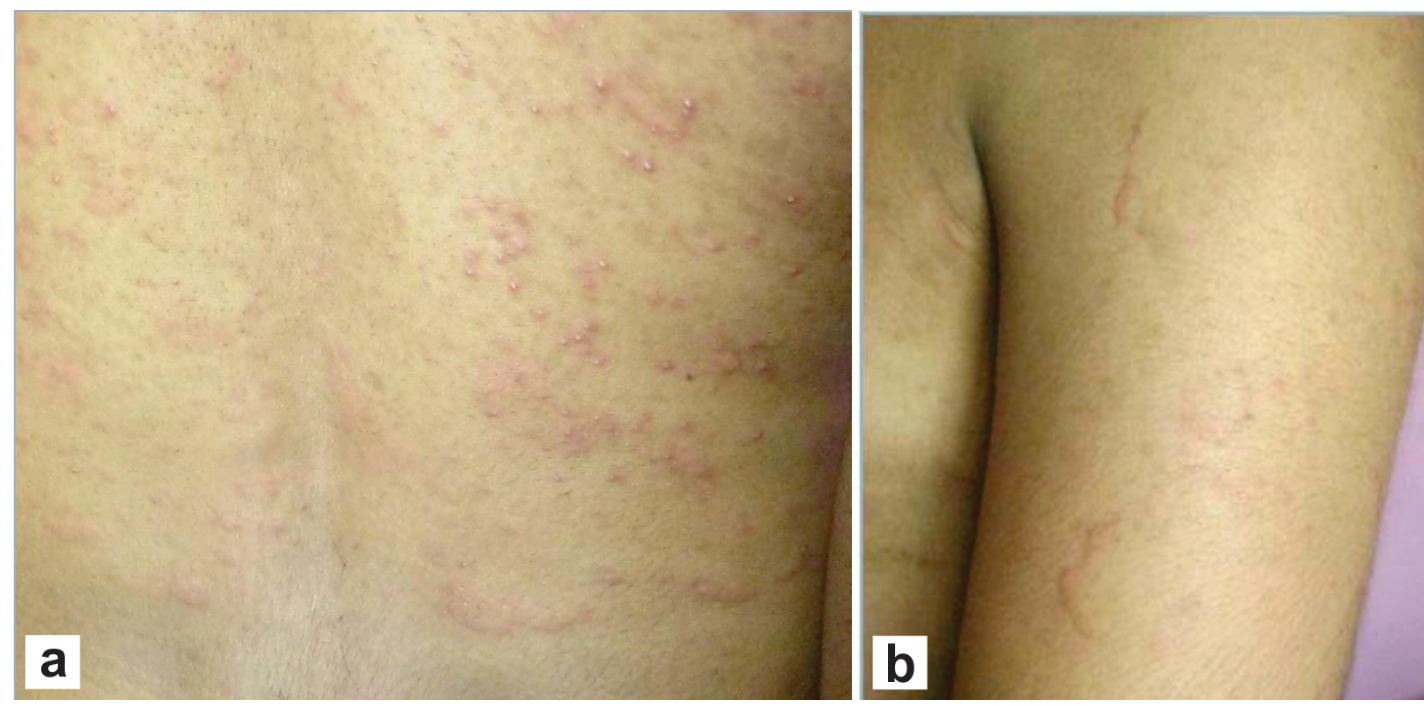

Figure 1 | Malassezia folliculitis: erythematous papules and pustules with urticarial wheals a) over trunk and b) left arm (Patient no. 3). 


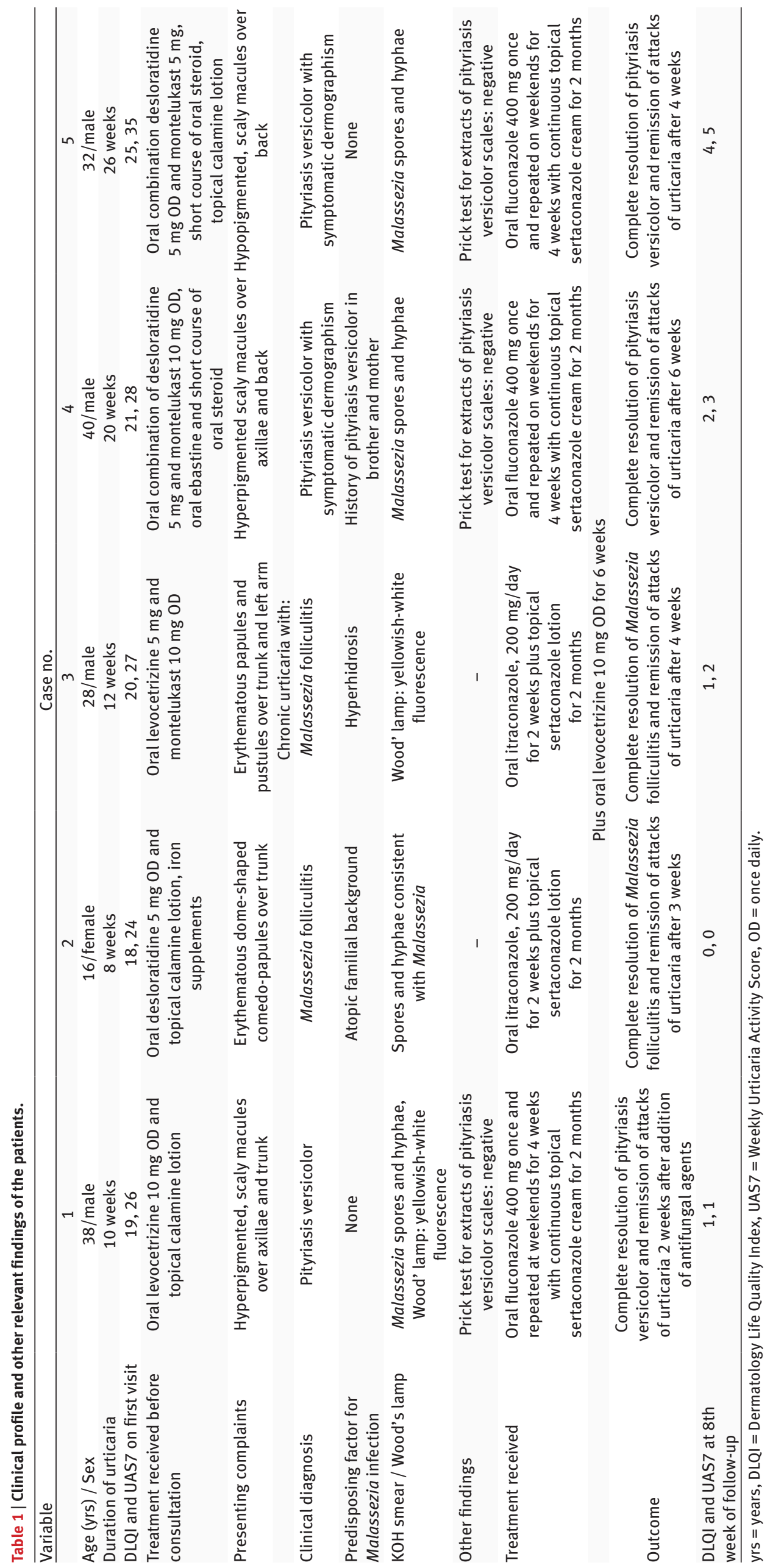




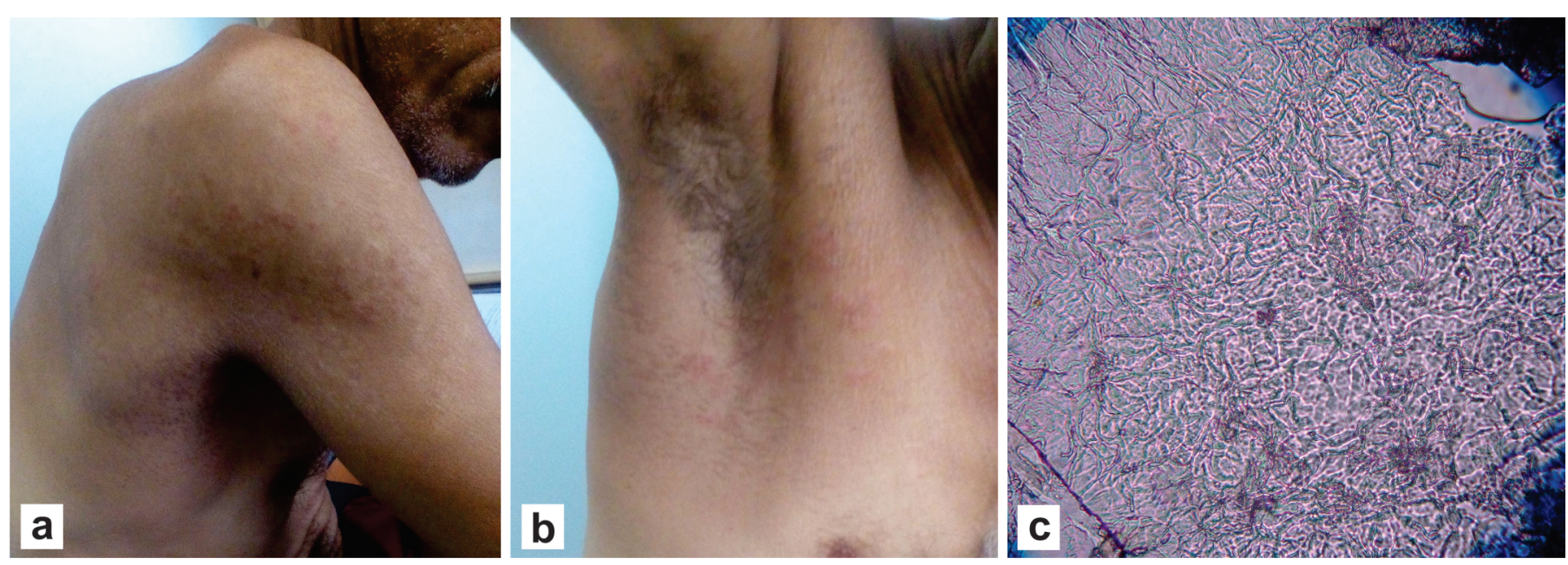

Figure 2 | Pityriasis versicolor: a) hyperpigmented scaly macules over the upper back and right shoulder; b) over the right axilla with urticarial weals; c) KOH examination of scales showing spores and hyphae (Patient no. 1).

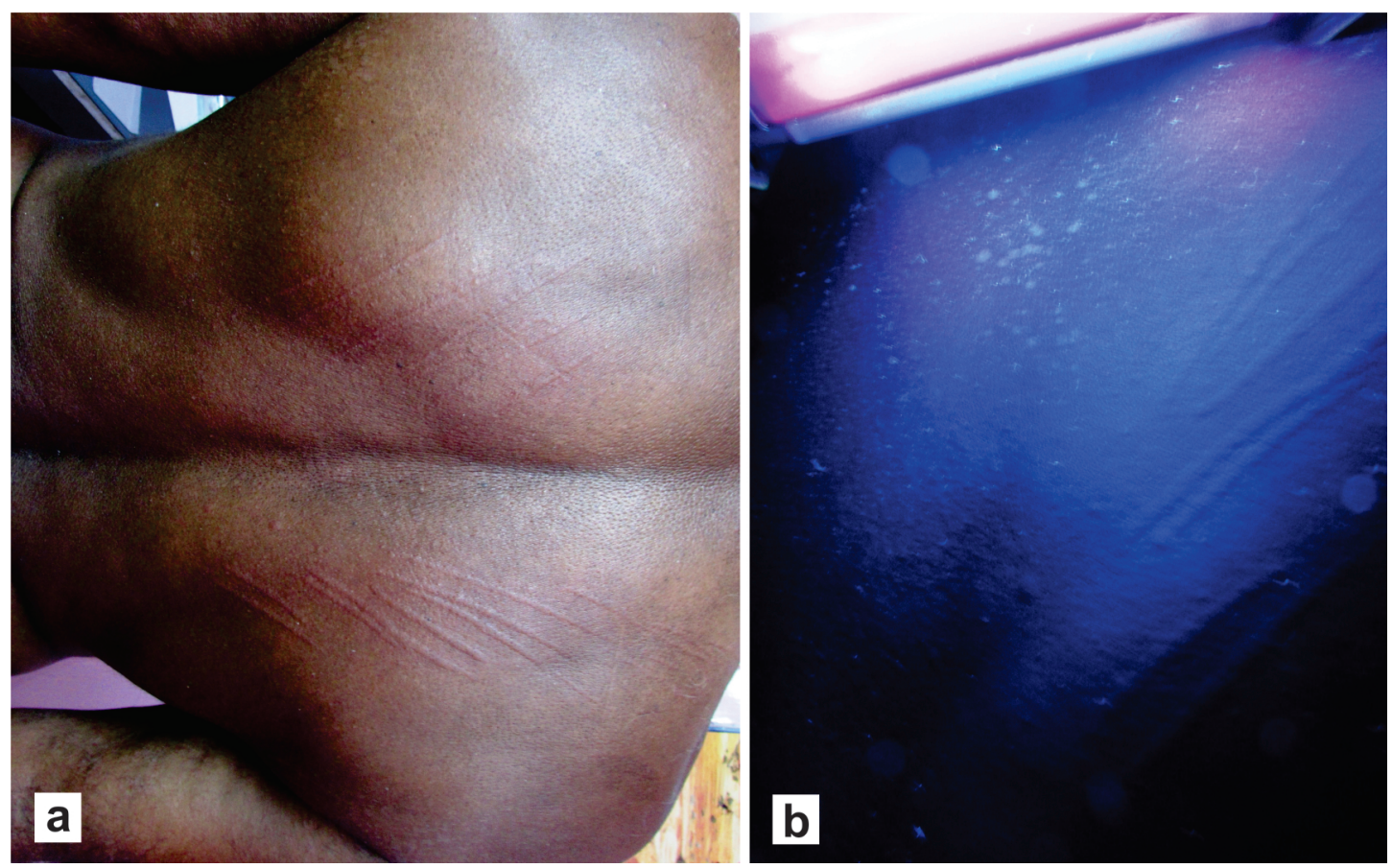

Figure 3 | Pityriasis versicolor: a) hypopigmented scaly macules over the left flank region with symptomatic dermographsim; b) Wood's lamp of the same showing bright yellow fluorescence (Patient no. 5).

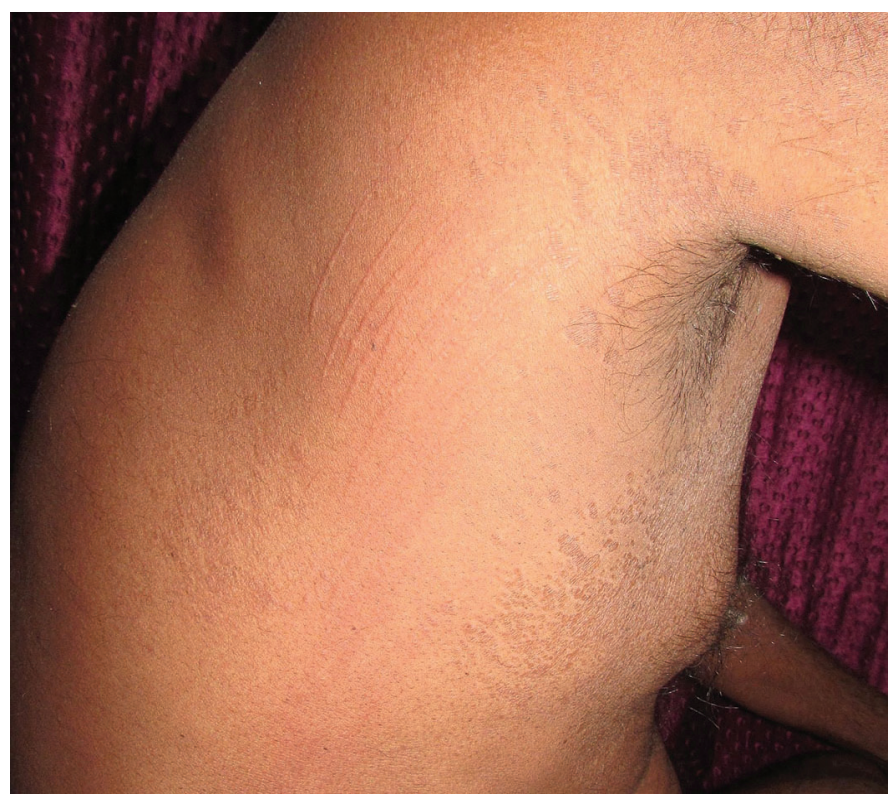

Figure 4 | Pityriasis versicolor: hyperpigmented scaly macules over the axilla and back with symptomatic dermographsim (Patient no. 4).

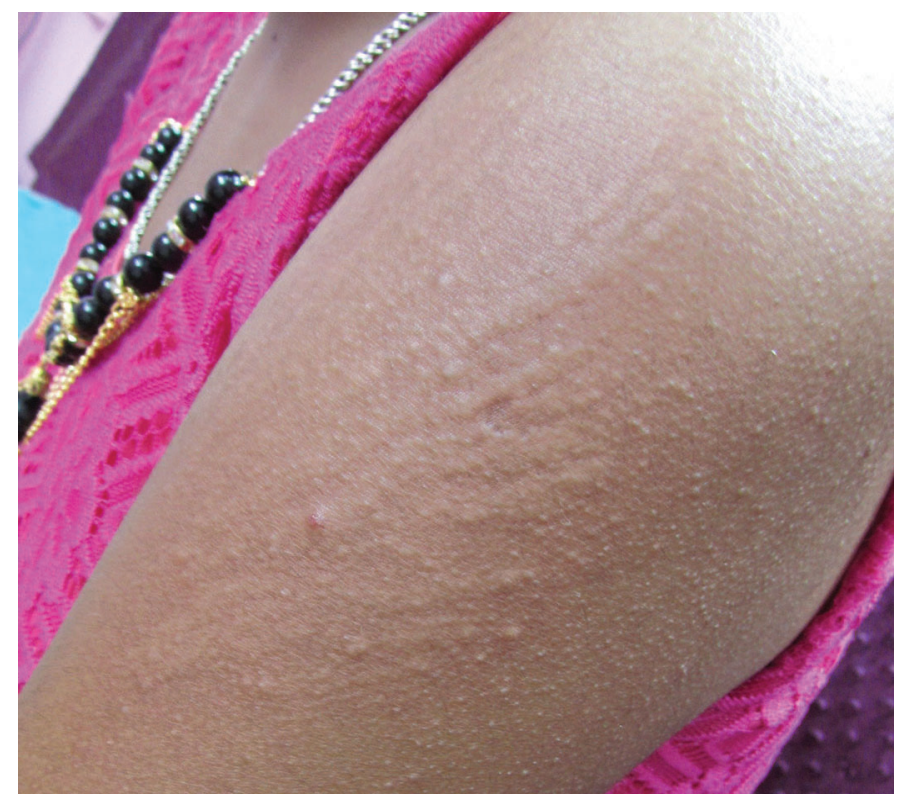

Figure 5 | Malassezia folliculitis over the left arm with transient edematous plaques suggestive of urticaria (Patient no. 2). 


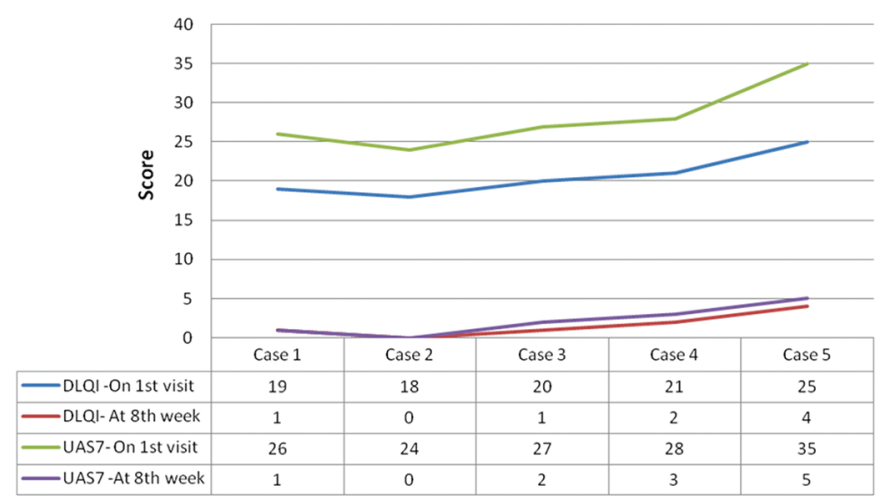

Figure 6 | Graphic description of DLQI and UAS-7 scores on first visit and at 8th week showing a sharp decline in DLQI and UAS-7 scores at the 8th week after the addition of antifungal therapy. DLQI = Dermatology Life Quality Index, UAS7 = Weekly Urticaria Activity Score.

All patients had coexisting Malassezia infection (either in the form of pityriasis versicolor or Malassezia folliculitis), which was confirmed by Wood's lamp and 10\% potassium hydroxide $(\mathrm{KOH})$ mount. They were treated with specific therapy directed against Malassezia with the addition of a systemic antihistaminic. After 6 weeks of therapy, all of the patients experienced remission of attacks of urticaria in addition to the elimination of Malassezia infection. None of the patients required further treatment with antihistaminics for the next 6 months of follow-up, and all of them remained symptom free with much improved quality of life.

\section{Discussion}

Almost $50 \%$ of cases of CSU are idiopathic and up to $31 \%$ of cases are associated with infections $(1,3)$. An association between CSU and occult infection has been proposed, but with little evidence. Microorganisms such as Helicobacter pylori and Streptococcus, Staphylococcus, and Yersinia infections have been recognized as the cause of urticaria (1). Most reported infections in CSU are related to the gastrointestinal tract, and to the dental and ear, nose, and throat region (1). Fungi implicated in CSU are Candida spp. and dermatophytes $(3,4)$. Hiragun et al. (6) demonstrated elevated levels of IgE against MGL_1304, a protein component of Malassezia contained in sweat, which is an important antigen for patients with atopic dermatitis and cholinergic urticaria.

Pathogenic forms of Malassezia are linked with pityriasis versicolor (PV), seborrhoeic dermatitis, and Malassezia folliculitis, and they have also been implicated in exacerbation of atopic dermatitis and psoriasis in certain patients. Because of their affinity to lipids, sebum-rich areas of the skin such as the face, scalp, and upper trunk are predominantly affected (7). Two metabolic pathways-phospholipase production and indole pigment synthesis-are associated with strains isolated from diseased human skin. The latter pathway produces potent indolic arylhydrocarbon receptor ligands such as indirubin and indolo carbazole, which potentially modify the epidermal cells' functions and thus play a major role in the pathogenesis of Malassezia infections (8). Indoles produced by Malassezia also downregulate the production of inflammatory mediators and antigen-presenting capacity (8).

The mechanism of causation of urticaria in our patients is unclear. The protein components of Malassezia include $67-\mathrm{kDa}$, $37^{-}$ $\mathrm{kDa}$, and $17-\mathrm{kDa}$. The $17-\mathrm{kDa}$ component (i.e., MGL_1304) has a high histamine-releasing property and it is also an important antigen for patients with atopic dermatitis and cholinergic urticaria. In contrast, the glycoprotein Malg46b component of $M$. globosa acts as a major antigen for IgE antibodies in patients with atopic dermatitis (9). It can be speculated that the protein components of Malassezia may act as haptens under specific environmental conditions of stimulation.

In all five patients, there was a temporal relationship of CSU with active Malassezia infection. Symptomatic dermographism was also seen in patients 4 and 5 . Although symptomatic dermographism is present in 4 to $5 \%$ of the normal population, its prevalence in CSU is reported to be $22 \%$ (10). Anti-histamine treatment was only partially effective and urticaria readily recurred after it was discontinued. There was significant deterioration of quality of life, as evident by high Dermatology Life Quality Index (DLQI) (11) and Weekly Urticaria Activity Scores (UAS7) $(12,13)$ on the first visit. Interestingly, 7 to 10 days after the addition of oral fluconazole/itraconazole and topical sertaconazole, a sharp decline in the DLQI and the UAS7 was observed (Fig. 6), which was maintained for the next 4 weeks, and all patients showed complete resolution and recurrences were stopped for next 6 months with drastic improvement in their quality of life.

Malassezia yeast is susceptible to topical and oral antifungal agents. Commonly used topical agents include azoles, hydroxypyridones, allylamines, benzylamines, tacrolimus, and pimecrolimus. We preferred oral itraconazole and fluconazole in patients with extensive involvement and in those with topical treatment failure (7).

Unfortunately, we could not identify the subspecies of Malassezia spp., evaluate the anti-Malassezia IgE antibodies, or perform a radioallergosorbent test, which may provide more insight into the link of urticaria with Malassezia. More detailed, multicentric, and larger studies on Malassezia infection and its specific association with CSU will be of more value.

The literature on urticaria in association with Malassezia is scant. To the best of our knowledge, only one study is available in PubMed, in which the authors found the carrier rates of Malassezia furfur to be significantly higher in a ship's crew suffering from urticaria than in normal control subjects (5).

\section{Conclusion}

We report five cases of chronic spontaneous urticaria associated with Malassezia infection. All of them showed significant remission of urticaria, especially after antifungal therapy directed against Malassezia infection in addition to oral levocetrizine, and they remained free of disease for the following 6 months.

\section{References}

1. Wedi B, Raap U, Wieczorek d, Kapp A. Urticaria and infections. Allergy Asthma Clin Immunol. 2009;5:10.

2. O'Donnell BF, Lawlor F, Simpson J, Morgan M, Greaves MW. The impact of chronic urticaria on the quality of life. Br J Dermatol 1997;136:197-201.

3. Ergon MC, Ilknur T, Yucesoy M, Ozkan S. Candida spp. colonization and serum anticandidal antibody levels in patients with chronic urticaria. Clin Exp Dermatol. 2007;32:740-3. 
4. Godse K, Zawar V. Chronic urticaria associated with tinea infection and success with antifungal therapy-a report of four cases. Int J Infect Dis 2010;Suppl 3:e364-5.

5. Tang XP, Zeng K, Chen GH, Bi LY, Fan LZ, Shao CF. Study of the association of Malassezia furfur with chronic urticaria among the ship crews. Di Yi Jun Yi Da Xue Xue Bao. 2003;23:870-2.

6. Hiragun M, Hiragun T, Ishii K, Suzuki H, Tanaka A, Yanase Y, et al. Elevated serum IgE against MGL_1304 in patients with atopic dermatitis and cholinergic urticaria. Allergol Int. 2014;63:83-93.

7. Gupta A, Batra R, Bluhm R, Boekhout T, Dawson TL. Skin diseases associated with Malassezia species. J Am Acad Dermatol. 2004;51:785-98.

8. Elegraki A, Cafarchia C, Gaitanis G, latta R, Boekhout T. Malassezia infections in humans and animals: pathophysiology, detection, and treatment. PloS Pathog. 2015;11:e1004523.

9. Kanbe T, Koyama T. Atopic dermatitis and Malassezia species: a study of antigenic components of Malassezia species for immunoglobulin E of patients with atopic dermatitis. Nihon Ishinkin Gakkai Zasshi. 2003;44:71-5.
10. Soter NA, Kaplan AP. Urticaria and angioedema. In: Freedberg IM, Eisen AZ, Wolff K, Austen KF, Goldsmith LA, Katz SI, editors. Fitzpatrick's Dermatology in general medicine. 6th ed. New York: McGraw-Hill; 2003. p. 1129-43.

11. Mtynek A, Zalewska-Janowska A, Martus P, Staubach P, Zuberbier T, Maurer M. How to assess disease activity in patients with chronic urticaria? Allergy. 2008;63:777-80.

12. Finlay AY, Khan GK. Dermatology Life Quality Index (DLQI)-a simple practical measure for routine clinical use. Clin Exp Dermatol. 1994;19:210-6.

13. Stull D, McBride D, Gimenez-Arnau A, Grattan C, Khalil S, Balp M-M. Validation of chronic spontaneous/idiopathic urticaria (CSU/CIU) health states using weekly Urticaria Activity Score (UAS7) and Dermatology Life Quality Index (DLQI) [poster]. American Academy of Dermatology Annual Meeting, San Francisco, March 2015. RTI Health Solutions. 2015 [cited 2017 Oct 4]. Available from: https://www.rtihs.org/publications/validation-chronic-spontaneousidiopathicurticaria-csuciu-health-states-using-weekly. 